\title{
Antioxidant and acetylcholinesterase-inhibitory properties of long-term stored medicinal plants
}

\author{
Stephen O Amoo, Adeyemi O Aremu, Mack Moyo and Johannes Van Staden*
}

\begin{abstract}
Background: Medicinal plants are possible sources for future novel antioxidant compounds in food and pharmaceutical formulations. Recent attention on medicinal plants emanates from their long historical utilisation in folk medicine as well as their prophylactic properties. However, there is a dearth of scientific data on the efficacy and stability of the bioactive chemical constituents in medicinal plants after prolonged storage. This is a frequent problem in African Traditional Medicine.

Methods: The phytochemical, antioxidant and acetylcholinesterase-inhibitory properties of 21 medicinal plants were evaluated after long-term storage of 12 or 16 years using standard in vitro methods in comparison to freshly harvested materials.

Results: The total phenolic content of Artemisia afra, Clausena anisata, Cussonia spicata, Leonotis intermedia and Spirostachys africana were significantly higher in stored compared to fresh materials. The flavonoid content were also significantly higher in stored A. afra, C. anisata, C. spicata, L. intermedia, Olea europea and Tetradenia riparia materials. With the exception of Ekebergia capensis and L. intermedia, there were no significant differences between the antioxidant activities of stored and fresh plant materials as measured in the $\beta$-carotene-linoleic acid model system. Similarly, the $\mathrm{EC}_{50}$ values based on the 2,2-diphenyl-1-picrylhydrazyl (DPPH) free radical scavenging assay were generally lower for stored than fresh material. Percentage inhibition of acetylcholinesterase was generally similar for both stored and fresh plant material. Stored plant material of Tetradenia riparia and Trichilia dregeana exhibited significantly higher AChE inhibition than the fresh material.
\end{abstract}

Conclusions: The current study presents evidence that medicinal plants can retain their biological activity after prolonged storage under dark conditions at room temperature. The high antioxidant activities of stable bioactive compounds in these medicinal plants offer interesting prospects for the identification of novel principles for application in food and pharmaceutical formulations.

Keywords: Antioxidants, Acetylcholinesterase inhibition, Long-term storage, Medicinal plants, Radical scavenging activity

\section{Background}

The detrimental effects of oxidative stress to human tissues and cells caused by reactive oxygen species (ROS) arising from aging and disease pathogenesis is well documented. Though the human body has inherent antioxidative mechanisms to counteract the damaging effects of free radicals, there is often a need to use dietary and/or medicinal antioxidant supplements, particularly during instances of disease attack. An imbalance between ROS

\footnotetext{
* Correspondence: rcpgd@ukzn.ac.za

Research Centre for Plant Growth and Development, School of Life Sciences, University of KwaZulu-Natal Pietermaritzburg, Private Bag X01, Scottsville 3209, South Africa
}

(c) 2012 Amoo et al.; licensee BioMed Central Ltd. This is an Open Access article distributed under the terms of the Creative Commons Attribution License (http://creativecommons.org/licenses/by/2.0), which permits unrestricted use, distribution, and reproduction in any medium, provided the original work is properly cited.

such as singlet oxygen, superoxide anion radical, hydroxyl radical and hydrogen peroxide, and the natural detoxification capacity of the body in favour of the oxidant molecules causes oxidative stress leading to cellular and DNA damage as well as oxidation of low-density lipoproteins $[1,2]$. Oxidative stress disorders caused by the actions of ROS are associated with many acute and chronic diseases such as inflammation and neurodegenerative conditions including Alzheimer's disease (AD) [3]. Alzheimer's disease, an age-related neurological disorder, is characterised by progressive loss of cognitive ability primarily memory loss, leading to dementia. The main strategy in the clinical treatment of $\mathrm{AD}$ involves the maintenance of adequate 
levels of acetylcholine (ACh) at neurotransmission sites [4]. Thus, the inhibition of acetylcholinesterase (AChE) prevents the hydrolysis of ACh thereby maintaining normal memory function. The consumption of antioxidants is highly correlated with lower incidences of $\mathrm{AD}[5,6]$. As a result, the use of natural compounds with high levels of antioxidants has been proposed as an effective therapeutic approach for $\mathrm{AD}$ [5].

Against a background of growing concerns about the toxicity and side effects of many synthetic therapeutic agents, there has been a renewed interest globally, in the search for antioxidants and $\mathrm{AChE}$ inhibitory compounds from natural sources, particularly medicinal plants $[1,2,7-$ 14]. Medicinal plants have long been used to treat cognitive memory dysfunction symptoms $[4,5,15-19]$. The growing relevance of medicinal plants as possible sources for the discovery of novel antioxidant molecules is often based on their long historical utilisation in folk medicine, especially in developing countries. In addition, the recognised health benefits of medicinal plants emanate from their prophylactic properties [6]. Most notably, traditional practices in the Ayurvedic, Chinese and African medicinal systems are strongly based on prevention and the promotion of good health; hence plant extracts and herbal preparations are regularly consumed as rejuvenators, tonics and/ or nutritional supplements [8]. Traditional medicine practitioners and gatherers often store plants before they are eventually consumed. However, there is a dearth of scientific data on the stability and efficacy of the bioactive compounds in medicinal plants after prolonged storage. In the present study, 21 commonly used South African medicinal plants (Table 1) were investigated for their phytochemical, antioxidant and AChE-inhibitory properties after 12 or 16 years storage in comparison to freshly harvested material. These plants are used in traditional medicine to prevent and/or treat pain-related ailments and infections [20-23]. Fresh materials were harvested from the same locations and season as the stored materials $[21,23]$ to minimise any differences due to geographical and seasonal effects [24].

\section{Methods}

\section{Chemicals and reagents}

Acetylcholine iodide, AChE from electric eel (type VI-S lyophilized powder), $\beta$-carotene, 2,2-diphenyl-1-picrylhydrazyl (DPPH), 5,5-dithiobis-2-nitrobenzoic acid (DTNB), galanthamine, gallic acid, catechin and linoleic acid were obtained from Sigma-Aldrich (Steinheim, Germany); butylated hydroxytoluene (BHT) from BDH Chemicals Ltd. (Poole, England); and harpagoside from Extrasynthèse (France). All chemicals and reagents used were of analytical grade.

\section{Plant material and preparation of extracts}

Table 1 shows the scientific names, and voucher specimen numbers of the evaluated plant materials. Following oven-drying at $50{ }^{\circ} \mathrm{C}$, plant materials were stored at room temperature $\left(25{ }^{\circ} \mathrm{C}\right)$ in brown paper bags in the dark for 12 or 16 years. Fresh plant materials collected from the same locations and season as the stored ones were similarly oven-dried at $50{ }^{\circ} \mathrm{C}$. The plants were identified by $\mathrm{Dr}$ C. Potgieter and voucher specimens deposited in The Bews Herbarium, University of KwaZulu-Natal, Pietermaritzburg, South Africa.

Dried plant materials were ground to fine powders and extracted with $50 \%$ methanol at $20 \mathrm{ml} / \mathrm{g}$ in a sonication bath containing ice-cold water for $1 \mathrm{~h}$ for antioxidant and AChE assays. Extracts were then filtered through Whatman No. 1 filter paper, concentrated in vacuo at $40{ }^{\circ} \mathrm{C}$ and completely air-dried at room temperature in glass vials.

The extraction method described by Makkar [25] was used for phytochemical analysis. Dried plant materials, ground to fine powders $(0.2 \mathrm{~g})$, were extracted with $50 \%$ aqueous methanol $(10 \mathrm{ml})$ in a sonication bath containing ice-cold water for $20 \mathrm{~min}$. The extracts were then centrifuged at approximately $3000 \mathrm{U} / \mathrm{min}$ for $5 \mathrm{~min}$ using a Hettich Universal 120001 Centrifuge. The supernatants were collected and kept on ice for phytochemical analysis.

\section{Phytochemical analysis}

Total iridoid content of the plant material was quantified using the method described by Levieille and Wilson [26]. The calibration curve was plotted using harpagoside as the standard. Total iridoid content for each plant material was expressed in $\mu \mathrm{g}$ harpagoside equivalents (HE) per g dry weight (DW).

For the determination of total phenolic content, the Folin \& Ciocalteu [27] method was used with slight modifications [28]. Gallic acid was used as the standard for plotting the calibration curve. Total phenolic content was expressed in mg gallic acid equivalents (GAE) per g DW.

The flavonoid content of the plant materials were quantified using the aluminium chloride colorimetric method [29]. Catechin was used as a standard for the calibration curve. Flavonoid content was expressed in mg catechin equivalents (CE) per g DW.

The butanol-HCl method [25] was used to quantify condensed tannin (proanthocyanidin) content of the plant materials. Condensed tannins (\% in dry matter) were expressed as leucocyanidin equivalents were calculated using the formula:

$$
\begin{aligned}
& \text { Condensed tannins }(\% \text { drymatter }) \\
& =\left(\frac{A_{550 \mathrm{~nm}} \times 78.26 \times \text { Dilutionfactor }}{\% \text { drymatter }}\right) \times 100
\end{aligned}
$$

where $A_{550 \mathrm{~nm}}$ is the absorbance of the sample at $550 \mathrm{~nm}$. The formula assumes that the effective $E_{550}^{1 \%}$ of leucocyanidin is 460 [30]. 
Table 1 Effect of long-term storage on the total iridoid, phenolic and flavonoid contents of 21 South African medicinal plants

\begin{tabular}{|c|c|c|c|c|c|c|c|c|c|}
\hline \multirow[t]{2}{*}{ Plant name } & \multirow[t]{2}{*}{ Family } & \multicolumn{2}{|c|}{ Voucher number Plant part(s) } & \multicolumn{2}{|c|}{ Total iridoids $(\mu \mathrm{g} \mathrm{HE} / \mathrm{g} \mathrm{DW})$} & \multicolumn{2}{|c|}{ Total phenolics (mg GAE/g DW) } & \multicolumn{2}{|c|}{ Total flavonoids (mg CE/g DW) } \\
\hline & & & & Stored & Fresh & Stored & Fresh & Stored & Fresh \\
\hline Acokanthera oppositifolia (Lam.) Codd ${ }^{\delta}$ & Apocynaceae & A. Aremu $1 \mathrm{NU}$ & Roots & $264.6 \pm 4.82 * *$ & $134.5 \pm 5.51$ & $7.5 \pm 0.37 *$ & $9.3 \pm 0.44$ & $4.8 \pm 0.12 *$ & $5.4 \pm 0.17$ \\
\hline Artemisia afra Jacq. ex Willd" & Asteraceae & S. Amoo $15 \mathrm{NU}$ & Aerial parts & $356.9 \pm 22.72 \mathrm{~ns}$ & $341.7 \pm 19.97$ & $28.5 \pm 1.15 \mathrm{~ns}$ & $25.8 \pm 0.03$ & $18.3 \pm 0.65 \mathrm{~ns}$ & $16.7 \pm 0.34$ \\
\hline Artemisia afra Jacq. ex Willd ${ }^{\delta}$ & Asteraceae & S. Amoo $15 \mathrm{NU}$ & Aerial parts & $195.1 \pm 63.35 \mathrm{~ns}$ & $341.7 \pm 19.97$ & $34.7 \pm 1.79 * *$ & $25.8 \pm 0.03$ & $19.7 \pm 0.87 *$ & $16.7 \pm 0.34$ \\
\hline Buddleja salviifolia (L.) Lam & Buddlejaceae & S. Amoo $16 \mathrm{NU}$ & Leaves & $60.8 \pm 15.84 * *$ & $409.9 \pm 13.77$ & $9.0 \pm 0.36^{* * *}$ & $20.0 \pm 0.81$ & $6.6 \pm 0.28 * * *$ & $14.6 \pm 0.32$ \\
\hline Buddleja salviifolia (L.) Lam & Buddlejaceae & S. Amoo $16 \mathrm{NU}$ & Twigs & $111.1 \pm 9.64 * *$ & $400.3 \pm 27.54$ & $8.3 \pm 0.25 * * *$ & $11.1 \pm 0.24$ & $5.0 \pm 0.25 *$ & $5.9 \pm 0.11$ \\
\hline Clausena anisata (Willd.) Hook. F. ex Benth" & Rutaceae & S. Amoo $18 \mathrm{NU}$ & Leaves \& Twigs & $3019.6 \pm 63.35 \mathrm{~ns}$ & $3264.7 \pm 96.40$ & $31.3 \pm 0.05 *$ & $28.1 \pm 0.99$ & $11.7 \pm 0.17 * *$ & $7.6 \pm 0.20$ \\
\hline Cussonia spicata Thunb. ${ }^{\#}$ & Araliaceae & S. Amoo $09 \mathrm{NU}$ & Leaves & $82.8 \pm 39.25 \mathrm{~ns}$ & $38.8 \pm 11.71$ & $11.4 \pm 0.16 * *$ & $7.6 \pm 0.69$ & $9.1 \pm 0.53 * * *$ & $3.4 \pm 0.27$ \\
\hline Dombeya rotundifolia Hochst. ${ }^{\#}$ & Malvaceae & S. Amoo $11 \mathrm{NU}$ & Leaves & $7076.6 \pm 177.64 * *$ & $9499.6 \pm 117.75$ & $45.3 \pm 0.89 \mathrm{~ns}$ & $47.3 \pm 1.94$ & $29.7 \pm 3.05 \mathrm{~ns}$ & $35.4 \pm 0.87$ \\
\hline Ekebergia capensis Sparrm $\delta$ & Meliaceae & S. Amoo $23 \mathrm{NU}$ & Leaves \& Twigs & $547.6 \pm 22.03 * * *$ & $2221.5 \pm 53.02$ & $31.7 \pm 1.29 * * *$ & $44.9 \pm 0.78$ & $22.8 \pm 1.25 \mathrm{~ns}$ & $26.0 \pm 0.29$ \\
\hline Leonotis intermedia Lindl..$^{\delta}$ & Lamiaceae & S. Amoo $08 \mathrm{NU}$ & Leaves & $56.0 \pm 1.38 *$ & $72.5 \pm 2.75$ & $15.1 \pm 0.57 * *$ & $11.6 \pm 0.23$ & $12.1 \pm 0.38 * * *$ & $6.8 \pm 0.10$ \\
\hline Leonotis leonurus (L.) R.Br. ${ }^{\delta}$ & Lamiaceae & S. Amoo $12 \mathrm{NU}$ & Leaves & $51.8 \pm 1.38 \mathrm{~ns}$ & $171.0 \pm 30.99$ & $10.5 \pm 0.22 * * *$ & $18.2 \pm 0.76$ & $6.6 \pm 0.23 * * *$ & $10.3 \pm 0.01$ \\
\hline Merwilla plumbea (Lindl.) Septa ${ }^{\delta}$ & Hyacinthaceae & S. Amoo $21 \mathrm{NU}$ & Bulbs & $64.2 \pm 8.26 \mathrm{~ns}$ & $207.5 \pm 75.74$ & $7.8 \pm 0.29 * *$ & $9.8 \pm 0.25$ & $1.4 \pm 0.09 \mathrm{~ns}$ & $1.7 \pm 0.37$ \\
\hline Ocotea bullata (Burch.) Baill. ${ }^{\delta}$ & Lauraceae & S. Amoo $13 \mathrm{NU}$ & Bark & $3060.9 \pm 121.19 * *$ & $6112.6 \pm 207.95$ & $32.7 \pm 0.82 * *$ & $46.4 \pm 2.00$ & $18.4 \pm 0.62 * * *$ & $26.8 \pm 0.50$ \\
\hline Olea europaea L." & Oleaceae & S. Amoo $14 \mathrm{NU}$ & Leaves & $0 \mathrm{~ns}$ & $283.2 \pm 79.87$ & $17.2 \pm 0.41 *$ & $18.7 \pm 0.06$ & $13.1 \pm 0.31 * * *$ & $9.7 \pm 0.28$ \\
\hline Pittosporum viridiflorum Sims ${ }^{\#}$ & Pittosporaceae & S. Amoo $24 \mathrm{NU}$ & Leaves \& Twigs & $63.6 \pm 8.95 \mathrm{~ns}$ & $194.4 \pm 65.41$ & $10.6 \pm 0.20 * * *$ & $26.0 \pm 0.91$ & $5.3 \pm 0.12^{* * *}$ & $15.6 \pm 0.22$ \\
\hline Plumbago auriculata Lam. ${ }^{\delta}$ & Plumbaginaceae & S. Amoo $06 \mathrm{NU}$ & Leaves & $9.8 \pm 7.57 * *$ & $521.4 \pm 50.95$ & $7.6 \pm 0.66^{* * *}$ & $15.0 \pm 0.46$ & $1.3 \pm 0.15 * *$ & $5.5 \pm 0.64$ \\
\hline Protorhus longifolia (Bernh.) Engl. ${ }^{\delta}$ & Anacardiaceae & S. Amoo $19 \mathrm{NU}$ & Leaves & $1034.4 \pm 47.51 * *$ & $7787.2 \pm 290.57$ & $51.8 \pm 1.27 * * *$ & $114.4 \pm 7.83$ & $10.1 \pm 0.65 * * *$ & $18.3 \pm 0.10$ \\
\hline Solanum mauritianum Scop. ${ }^{\delta}$ & Solanaceae & S. Amoo $07 \mathrm{NU}$ & Leaves & $71.1 \pm 6.89 *$ & $14.0 \pm 11.71$ & $8.0 \pm 0.11 * * *$ & $13.9 \pm 0.24$ & $2.0 \pm 0.21 \mathrm{~ns}$ & $1.5 \pm 0.05$ \\
\hline Spirostachys africana Sond." & Euphorbiaceae & S. Amoo $26 \mathrm{NU}$ & Leaves \& Twigs & $553.8 \pm 3.44 \mathrm{~ns}$ & $527.6 \pm 11.71$ & $86.2 \pm 1.91 * *$ & $69.1 \pm 2.13$ & $8.5 \pm 0.09 * * *$ & $26.7 \pm 0.57$ \\
\hline Synadenium copulare (Boiss.) L.C. Wheeler ${ }^{\delta}$ & Euphorbiaceae & S. Amoo $25 \mathrm{NU}$ & Leaves & $11.9 \pm 11.02 \mathrm{~ns}$ & $273.6 \pm 71.61$ & $8.5 \pm 0.37^{* * *}$ & $15.2 \pm 0.33$ & $4.2 \pm 0.15 \mathrm{~ns}$ & $4.3 \pm 0.15$ \\
\hline Tetradenia riparia (Hochst.) Codd ${ }^{\delta}$ & Lamiaceae & S. Amoo $20 \mathrm{NU}$ & Leaves & $46.3 \pm 9.64{ }^{*}$ & 0 & $6.1 \pm 0.20 \mathrm{~ns}$ & $7.2 \pm 0.38$ & $2.7 \pm 0.08 * * *$ & $1.5 \pm 0.02$ \\
\hline Trichilia dregeana Sond. ${ }^{\delta}$ & Meliaceae & S. Amoo $22 \mathrm{NU}$ & Leaves \& Twigs & $431.9 \pm 16.53 \mathrm{~ns}$ & $412.0 \pm 50.27$ & $34.4 \pm 10.26 \mathrm{~ns}$ & $32.6 \pm 1.17$ & $8.7 \pm 0.61 * * *$ & $20.2 \pm 0.19$ \\
\hline Ziziphus mucronata Willd." & Rhamnaceae & S. Amoo $17 \mathrm{NU}$ & Leaves & $314.2 \pm 37.87 \mathrm{~ns}$ & $412.7 \pm 22.03$ & $23.6 \pm 1.61 * *$ & $33.4 \pm 0.62$ & $7.1 \pm 0.10^{* * *}$ & $9.0 \pm 0.09$ \\
\hline Ziziphus mucronata Willd. ${ }^{\delta}$ & Rhamnaceae & S. Amoo $17 \mathrm{NU}$ & Leaves & $90.4 \pm 1.38 * *$ & $412.7 \pm 22.03$ & $19.7 \pm 0.42 * * *$ & $33.4 \pm 0.62$ & $6.9 \pm 0.34 * *$ & $9.0 \pm 0.09$ \\
\hline
\end{tabular}

ns $=$ not significant; $P=0.05\left(^{*}\right) ; P=0.01\left(^{* *}\right) ; P=0.001\left(^{* * *}\right)$.

$\mathrm{HE}=$ harpagoside equivalents; $\mathrm{GAE}=$ gallic acid equivalents; $\mathrm{CE}=$ catechin equivalents.

$\delta=$ Voucher number of plant material stored for 16 years was as described by Jäger et al. (1996); ${ }^{\#}=$ Voucher number of plant material stored for 12 years was as described by McGaw et al. (2000).

Merwilla plumbea (Lindl.) Speta was formerly known as Scilla natalensis Planch. 
Free gallic acid and gallotannin contents were evaluated using the rhodanine assay [25,31]. The calibration curves were plotted using gallic acid as a standard. Free gallic acid and gallotannin contents were expressed in $\mu \mathrm{g}$ GAE per g DW.

\section{Antioxidant activity DPPH free radical scavenging activity}

The DPPH assay [32] was used to evaluate the free radical scavenging activity of the plant extracts. Methanol was used as a negative control while ascorbic acid and BHT were used as positive controls. Any absorbance due to extract colour was removed by including a background solution with methanol in place of DPPH solution for each extract. Each sample was evaluated in triplicate. The radical scavenging activity (RSA) was calculated using the equation:

$$
R S A(\%)=\left[1-\left(\frac{A_{\text {extract }}-A_{\text {background }}}{A_{\text {control }}}\right)\right] \times 100
$$

where $A_{\text {extract }}, A_{\text {background }}$ and $A_{\text {control }}$ are the absorbance readings of the extract, background solution and negative control, respectively at $517 \mathrm{~nm}$. The $\mathrm{EC}_{50}$, which is the extract concentration required to scavenge $50 \%$ of DPPH free radical, was determined for each extract. Antioxidant activity index (AAI) for each extract was calculated using the equation [33]:

$$
A A I=\frac{\text { FinalDPPH concentration }}{E C_{50}}
$$

\section{$\beta$-Carotene-linoleic acid model system}

The assay was done following the method described by Moyo et al. [34]. Methanol and BHT were used as negative and positive controls, respectively. Each sample was prepared in triplicate. The plant extracts and BHT were evaluated at a final assay concentration of $200 \mu \mathrm{g} / \mathrm{ml}$. Antioxidant activity (\%), measured at $t=120 \mathrm{~min}$, was calculated using the following equations:

$$
\begin{gathered}
\text { Rate of } \beta-\text { carotene bleaching }=\operatorname{In}\left(\frac{A_{t=0}}{A_{t=t}}\right) \times \frac{1}{t} \\
\text { Antioxidant activity }(\%)=\left(\frac{\text { Rcontorl }- \text { Rsample }}{\text { Rcontrol }}\right) \times 100
\end{gathered}
$$

where $A_{t}=0$ is the initial absorbance at $t=0 \mathrm{~min}, A_{t}=t$ is the absorbance at time $t=120 \mathrm{~min}, \mathrm{t}=120 \mathrm{~min}$ and $R$ is the rate of $\beta$-carotene bleaching.

\section{Acetylcholinesterase inhibitory activity}

The AChE assay was performed using the colorimetric method [35]. Each extract was evaluated in triplicate at a final assay concentration of $1.0 \mathrm{mg} / \mathrm{ml}$. Galanthamine at a final assay concentration of $20 \mu \mathrm{M}$ was used as a positive control. The rate of reaction was calculated for each of the plant extracts, the blank (methanol) and positive control (galanthamine). The percentage inhibition by each plant extract was calculated using the formula:

AChE inhibition $(\%)=\left(1-\frac{\text { Sample reaction rate }}{\text { Blank reaction rate }}\right) \times 100$

\section{Data analysis}

The levels of significant difference between the mean values of stored and fresh plant materials were determined using the $t$-test (SigmaPlot version 8.0). Regression analysis and the determination of $\mathrm{EC}_{50}$ values were done using GraphPad Prism software (version 4.03).

\section{Results and discussion \\ Phytochemical analysis}

The effects of long-term storage on the total iridoid, phenolic and flavonoid content of the plant materials evaluated are presented in Table 1 . Of the 21 fresh and stored plant materials evaluated, the levels of total iridoid present in nine plants were significantly higher in fresh compared to the stored plant materials. The total iridoid contents of stored materials in Acokanthera oppositifolia, Solanum mauritanum and Tetradenia riparia were significantly higher than those of fresh ones. There was no significant difference between the iridoid content of fresh and stored plant materials in approximately $50 \%$ of the evaluated plants.

The total phenolic contents of Artemisia afra, Clausena anisata, Cussonia spicata, Leonotis intermedia and Spirostachys africana stored materials were significantly higher than in freshly collected material. With the exceptions of $A$. afra, $D$. rotundifolia, $T$. riparia and T. dregeana (where there was no significant difference between the stored and fresh materials), the phenolic contents of the remaining 15 fresh plant materials were significantly higher than in the stored material. Similarly, a comparison of fresh material and herbarium specimens of three Quillaja species revealed non-significant differences in their phenolic constituents [36]. Remarkably, one of the tested herbarium specimens in the BateSmith [36] study was 100 years old.

The flavonoid content was significantly higher in stored A. afra, C. anisata, C. spicata, L. intermedia, T. riparia and Olea europea materials when compared to their corresponding fresh materials. It is noteworthy that the stored materials of the former four species had higher total phenolic contents than their fresh materials perhaps due to their higher flavonoid content compared to the fresh materials. Higher flavonoid contents were observed in 12 fresh plant materials when compared to their respective stored materials. Previous studies 
Table 2 Effect of long-term storage on the condensed tannin, free gallic acid and gallotannin contents of 21 South African medicinal plants

\begin{tabular}{|c|c|c|c|c|c|c|c|}
\hline \multirow[t]{2}{*}{ Plant name } & \multirow[t]{2}{*}{ Plant part(s) } & \multicolumn{2}{|c|}{ Condensed tannins ( $\%$ in dry matter) } & \multicolumn{2}{|c|}{ Free gallic acid ( $\mu \mathrm{g}$ GAE/g DW) } & \multicolumn{2}{|c|}{ Gallotannins ( $\mu \mathrm{g}$ GAE/g DW) } \\
\hline & & Stored & Fresh & Stored & Fresh & Stored & Fresh \\
\hline Acokanthera oppositifolia ${ }^{\delta}$ & Roots & 0 & 0 & $2.996 \pm 2.9963 \mathrm{~ns}$ & $1.284 \pm 1.2841$ & $32.960 \pm 0.4281 *$ & $60.355 \pm 6.4207$ \\
\hline Artemisia afra $^{\#}$ & Aerial parts & $0.078 \pm 0.0005^{* * *}$ & 0 & 0 & 0 & $76.621 \pm 6.4207$ ns & $97.167 \pm 10.7012$ \\
\hline Artemisia afra ${ }^{\delta}$ & Aerial parts & $0.004 \pm 0.0002 *$ & 0 & 0 & 0 & $27.823 \pm 11.5573 *$ & $97.167 \pm 10.7012$ \\
\hline Buddleja salviifolia\# & Leaves & $0.011 \pm 0.0002 *$ & $0.056 \pm 0.0073$ & $29.535 \pm 20.1183 \mathrm{~ns}$ & 0 & $80.720 \pm 17.0557 \mathrm{~ns}$ & $41.949 \pm 1.7122$ \\
\hline Buddleja salviifolia" & Twigs & $0.017 \pm 0.0047 \mathrm{~ns}$ & $0.005 \pm 0.0050$ & $17.122 \pm 3.4244 \mathrm{~ns}$ & $8.133 \pm 5.5646$ & $14.982 \pm 8.1329 \mathrm{~ns}$ & $38.096 \pm 17.5500$ \\
\hline Clausena anisata ${ }^{\#}$ & Leaves \& Twigs & $1.394 \pm 0.0318 \mathrm{~ns}$ & $1.329 \pm 0.0159$ & 0 & 0 & $68.488 \pm 5.1366 * *$ & 0 \\
\hline Cussonia spicata\# & Leaves & $0.012 \pm 0.0016^{*}$ & 0 & $138.260 \pm 41.5208 \mathrm{~ns}$ & $12.842 \pm 12.8415$ & $397.377 \pm 55.8931 \mathrm{~ns}$ & $468.758 \pm 81.3346$ \\
\hline Dombeya rotundifolia\# & Leaves & $1.804 \pm 0.0116^{* *}$ & $0.973 \pm 0.0529$ & 0 & 0 & $41.949 \pm 19.6903 \mathrm{~ns}$ & 0 \\
\hline Ekebergia capensis $^{\delta}$ & Leaves \& Twigs & $0.654 \pm 0.0040 \mathrm{~ns}$ & $0.523 \pm 0.0706$ & 0 & 0 & $0 * *$ & $19.690 \pm 2.5683$ \\
\hline Leonotis intermedia ${ }^{\delta}$ & Leaves & $0.008 \pm 0.0007^{* *}$ & 0 & $0 \mathrm{~ns}$ & $3.424 \pm 3.4244$ & $17.550 \pm 5.5646 \mathrm{~ns}$ & $11.129 \pm 4.2805$ \\
\hline Leonotis leonurus ${ }^{\delta}$ & Leaves & $0.011 \pm 0.0002 * * *$ & 0 & $0 * * *$ & $47.085 \pm 1.7122$ & $24.827 \pm 0.8561 *$ & $5.565 \pm 3.8524$ \\
\hline Merwilla plumbea ${ }^{\delta}$ & Bulbs & 0 & 0 & $8.133 \pm 8.1329 \mathrm{~ns}$ & $23.971 \pm 7.7049$ & $167.367 \pm 13.2695$ & ND \\
\hline Ocotea bullata ${ }^{\delta}$ & Bark & $1.154 \pm 0.0162 * *$ & $0.699 \pm 0.0354$ & 0 & 0 & $68.060 \pm 8.9890 *$ & $14.982 \pm 7.2768$ \\
\hline Olea europaea\# & Leaves & $0.010 \pm 0.0019 *$ & 0 & 0 & 0 & $127.559 \pm 4.2805 \mathrm{~ns}$ & $121.566 \pm 1.7122$ \\
\hline Pittosporum viridiflorum ${ }^{\#}$ & Leaves \& Twigs & 0 & 0 & $5.565 \pm 5.5646 \mathrm{~ns}$ & 0 & $75.337 \pm 0.8561$ ns & $66.776 \pm 6.8488$ \\
\hline Plumbago auriculata ${ }^{\delta}$ & Leaves & $0.003 \pm 0.0011 * *$ & $0.024 \pm 0.0013$ & $3.852 \pm 3.8524 \mathrm{~ns}$ & 0 & $20.118 \pm 5.5646 \mathrm{~ns}$ & $4.7085 \pm 4.7085$ \\
\hline Protorhus longifolia ${ }^{\delta}$ & Leaves & $0.400 \pm 0.0127 \mathrm{~ns}$ & $0.724 \pm 0.0885$ & $2398.787 \pm 112.1485 \mathrm{~ns}$ & $1901.394 \pm 137.8318$ & $2726.245 \pm 615.9627 \mathrm{~ns}$ & $4039.926 \pm 1368.0443$ \\
\hline Solanum mauritianum ${ }^{\delta}$ & Leaves & $0.013 \pm 0.0013 \mathrm{~ns}$ & $0.005 \pm 0.005$ & $32.103 \pm 4.7085 \mathrm{~ns}$ & $23.971 \pm 0.8561$ & $183.047 \pm 75.6858$ & ND \\
\hline Spirostachys africana ${ }^{\#}$ & Leaves \& Twigs & $0.348 \pm 0.0083 \mathrm{~ns}$ & $0.365 \pm 0.0311$ & $1107.363 \pm 228.1501 *$ & 0 & $2445.016 \pm 118.1414 * *$ & $16.266 \pm 16.2659$ \\
\hline Synadenium cupulare & Leaves & $0.010 \pm 0.0002 * *$ & $0.004 \pm 0.0004$ & $0 \mathrm{~ns}$ & $8.561 \pm 8.5610$ & $20.546 \pm 4.2805$ ns & $54.362 \pm 21.8305$ \\
\hline Tetradenia riparia ${ }^{\delta}$ & Leaves & $0.002 \pm 0.0004 \mathrm{~ns}$ & $0.005 \pm 0.0022$ & $0^{* * *}$ & $14.982 \pm 0.4280$ & $11.985 \pm 1.7122 \mathrm{~ns}$ & $22.259 \pm 13.6976$ \\
\hline Trichilia dregeana $^{\delta}$ & Leaves \& Twigs & $0.198 \pm 0.0099$ ns & $0.138 \pm 0.0148$ & $118.998 \pm 5.1366 * *$ & 0 & $442.603 \pm 65.0634 *$ & $13.270 \pm 1.2841$ \\
\hline Ziziphus mucronata" & Leaves & $0.008 \pm 0.0000^{* * *}$ & $0.046 \pm 0.0013$ & 0 & 0 & $38.953 \pm 12.4134 \mathrm{~ns}$ & $28.679 \pm 1.2842$ \\
\hline Ziziphus mucronata ${ }^{\delta}$ & Leaves & $0.077 \pm 0.0003 * *$ & $0.046 \pm 0.0013$ & 0 & 0 & $47.085 \pm 17.1220 \mathrm{~ns}$ & $28.679 \pm 1.2842$ \\
\hline
\end{tabular}

ns = not significant; $P=0.05(*) ; P=0.01(* *) ; P=0.001\left(^{* * *}\right) ; \mathrm{ND}=$ not determined; $\mathrm{GAE}=$ Gallic acid equivalents.

$\delta=$ Plant material stored for 16 years.

${ }^{\#}=$ Plant material stored for 12 years. 
comparing the phenolic constituents of some Dillenia species showed differences in the flavonoid profiles of fresh and herbarium materials as some flavonoids were not detected in the latter [37]. The results suggested that some flavonoids are easily oxidised during the drying process [37].

Table 2 presents the condensed tannin, free gallic acid and gallotannin contents of both the stored and fresh materials of plant species evaluated in this study. No condensed tannins were detected in both fresh and stored materials of A. oppositifolia, Pittosporum viridiflorum and Merwilla plumbea. With the exceptions of Buddleja salviifolia (leaves), Plumbago auriculata and Ziziphus mucronata, the condensed tannin content in the stored plant materials was either significantly higher or not different when compared to the fresh materials. Unlike the stored materials, no condensed tannins were detected in fresh material of $A$. afra, C. spicata, L. intermedia, Leonotis leonurus and $O$. europea. Among the 21 species evaluated, free gallic acid was detected in 15 fresh and/or stored plant materials. In most cases, there was no significant difference in the free gallic acid contents of the fresh materials when compared to the stored ones. With the exceptions of A. oppositifolia, A. afra and Ekebergia capensis, the gallotannin content of the stored plant materials was either higher or not significantly different when compared to the fresh ones. It has been shown that phytochemical constituents of medicinal plants, such as alkaloids, flavonoids, volatile oils and amino acids are sufficiently stable to even be detected in herbarium specimens [38]. However, based on the results of the present study, the degree of stability of phenolic compounds seems to be species dependent.

Table 3 Effect of long-term storage on the free radical scavenging activity of 21 South African medicinal plants

\begin{tabular}{|c|c|c|c|c|c|c|c|}
\hline \multirow[t]{2}{*}{ Plant species } & \multirow[t]{2}{*}{ Plant part } & \multicolumn{2}{|c|}{ Radical scavenging activity (\%) at $100 \mu \mathrm{g} / \mathrm{ml}$} & \multicolumn{2}{|c|}{$\mathrm{EC}_{50}(\mu \mathrm{g} / \mathrm{ml})$} & \multicolumn{2}{|c|}{ Antioxidant activity index } \\
\hline & & Stored & Fresh & Stored & Fresh & Stored & Fresh \\
\hline Acokanthera oppositifolia ${ }^{\delta}$ & Roots & $93.3 \pm 0.03 * *$ & $92.6 \pm 0.10$ & $26.8 \pm 2.43 *$ & $18.0 \pm 0.34$ & $0.7 \pm 0.06 * *$ & $1.1 \pm 0.02$ \\
\hline Artemisia afra & Aerial parts & $93.8 \pm 0.11 *$ & $92.7 \pm 0.34$ & $9.3 \pm 0.07^{* * *}$ & $12.4 \pm 0.15$ & $2.1 \pm 0.02 * * *$ & $1.6 \pm 0.02$ \\
\hline Artemisia afra $^{\delta}$ & Aerial parts & $94.0 \pm 0.07 *$ & $92.7 \pm 0.34$ & $6.8 \pm 0.50 * * *$ & $12.4 \pm 0.15$ & $2.9 \pm 0.21 * *$ & $1.6 \pm 0.02$ \\
\hline Buddleja salviifolia" & Leaves & $96.2 \pm 0.06 * * *$ & $93.0 \pm 0.40$ & $15.5 \pm 0.47^{* *}$ & $10.0 \pm 0.61$ & $1.3 \pm 0.04 * *$ & $2.0 \pm 0.12$ \\
\hline Buddleja salviifolia" & Twigs & $94.2 \pm 0.13 \mathrm{~ns}$ & $94.3 \pm 0.15$ & $17.2 \pm 0.32 \mathrm{~ns}$ & $17.5 \pm 0.40$ & $1.1 \pm 0.02 \mathrm{~ns}$ & $1.1 \pm 0.03$ \\
\hline Clausena anisata & Leaves \& Twigs & $70.8 \pm 0.28 \mathrm{~ns}$ & $72.6 \pm 6.21$ & $33.2 \pm 3.89 \mathrm{~ns}$ & $26.8 \pm 2.06$ & $0.6 \pm 0.07 \mathrm{~ns}$ & $0.7 \pm 0.06$ \\
\hline Cussonia spicata & Leaves & $93.7 \pm 0.07 * * *$ & $61.6 \pm 1.67$ & $14.3 \pm 0.22 * *$ & $43.6 \pm 5.73$ & $1.4 \pm 0.02^{* * *}$ & $0.5 \pm 0.07$ \\
\hline Dombeya rotundifolia ${ }^{\#}$ & Leaves & $96.5 \pm 0.56^{* *}$ & $93.6 \pm 0.27$ & $5.9 \pm 0.12 \mathrm{~ns}$ & $6.1 \pm 0.32$ & $3.3 \pm 0.07 \mathrm{~ns}$ & $3.2 \pm 0.16$ \\
\hline Ekebergia capensis ${ }^{\delta}$ & Leaves \& Twigs & $94.2 \pm 0.42 *$ & $92.8 \pm 0.30$ & $4.7 \pm 0.37^{* *}$ & $25.5 \pm 4.99$ & $4.3 \pm 0.32^{* * *}$ & $0.8 \pm 0.14$ \\
\hline Leonotis intermedia $\delta$ & Leaves & $93.3 \pm 0.09 *$ & $88.5 \pm 1.73$ & $10.6 \pm 0.37^{* * *}$ & $51.7 \pm 0.32$ & $1.9 \pm 0.06^{* * *}$ & $0.4 \pm 0.00$ \\
\hline Leonotis leonurus $^{\delta}$ & Leaves & $93.7 \pm 0.18 * *$ & $91.6 \pm 0.43$ & $16.8 \pm 0.06^{* * *}$ & $30.3 \pm 0.92$ & $1.2 \pm 0.00 * * *$ & $0.7 \pm 0.02$ \\
\hline Merwilla plumbea ${ }^{\delta}$ & Bulbs & $8.2 \pm 0.61 * *$ & $2.6 \pm 0.97$ & ND & ND & ND & ND \\
\hline Ocotea bullata & Bark & $95.0 \pm 0.25 * *$ & $93.8 \pm 0.02$ & $3.2 \pm 0.14 * *$ & $4.3 \pm 0.10$ & $6.3 \pm 0.28 * *$ & $4.6 \pm 0.11$ \\
\hline Olea europaea & Leaves & $94.9 \pm 0.20 * *$ & $93.2 \pm 0.09$ & $14.0 \pm 0.48^{* * *}$ & $20.0 \pm 0.16$ & $1.4 \pm 0.05^{* * *}$ & $1.0 \pm 0.01$ \\
\hline Pittosporum viridiflorum ${ }^{\#}$ & Leaves \& Twigs & $93.6 \pm 0.10 \mathrm{~ns}$ & $93.8 \pm 0.29$ & $17.9 \pm 0.25 \mathrm{~ns}$ & $17.5 \pm 0.27$ & $1.1 \pm 0.02 \mathrm{~ns}$ & $1.1 \pm 0.02$ \\
\hline Plumbago auriculata $^{\delta}$ & Leaves & $50.6 \pm 3.97 \mathrm{~ns}$ & $54.6 \pm 1.15$ & ND & ND & ND & ND \\
\hline Protorhus longifolia ${ }^{\delta}$ & Leaves & $95.8 \pm 0.24 * *$ & $97.3 \pm 0.21$ & $2.2 \pm 0.16 \mathrm{~ns}$ & $2.3 \pm 0.14$ & $9.1 \pm 0.71 \mathrm{~ns}$ & $8.5 \pm 0.49$ \\
\hline Solanum mauritianum $\delta$ & Leaves & $34.4 \pm 0.73^{* * *}$ & $19.8 \pm 1.53$ & ND & ND & ND & ND \\
\hline Spirostachys africana ${ }^{\#}$ & Leaves \& Twigs & $96.6 \pm 0.06^{* * *}$ & $91.8 \pm 0.34$ & $2.0 \pm 0.07 * * *$ & $14.4 \pm 0.58$ & $10.0 \pm 0.35 * * *$ & $1.4 \pm 0.06$ \\
\hline Synadenium cupulare ${ }^{\delta}$ & Leaves & $90.9 \pm 0.70 * * *$ & $46.0 \pm 5.30$ & $55.7 \pm 0.35$ & ND & $0.4 \pm 0.02$ & ND \\
\hline Tetradenia riparia ${ }^{\delta}$ & Leaves & $68.5 \pm 1.39 * * *$ & $23.8 \pm 2.44$ & $41.0 \pm 5.29$ & ND & $0.5 \pm 0.06$ & ND \\
\hline Trichilia dregeana ${ }^{\#}$ & Leaves \& Twigs & $95.8 \pm 0.46^{* *}$ & $92.3 \pm 0.16$ & $5.3 \pm 0.02 * * *$ & $14.6 \pm 0.24$ & $3.7 \pm 0.01 * * *$ & $1.3 \pm 0.02$ \\
\hline Ziziphus mucronata & Leaves & $90.7 \pm 0.42 \mathrm{~ns}$ & $89.0 \pm 2.20$ & $29.7 \pm 1.02 \mathrm{~ns}$ & $30.9 \pm 1.94$ & $0.7 \pm 0.02 \mathrm{~ns}$ & $0.6 \pm 0.04$ \\
\hline Ziziphus mucronata & Leaves & $91.1 \pm 0.18 \mathrm{~ns}$ & $89.0 \pm 2.20$ & $18.1 \pm 0.29 * *$ & $30.9 \pm 1.94$ & $1.1 \pm 0.02 * * *$ & $0.6 \pm 0.04$ \\
\hline Ascorbic acid & & $96.6 \pm 0.04$ & & $2.1 \pm 0.05$ & & $9.4 \pm 0.23$ & \\
\hline Butylated hydroxytoluene & & $93.2 \pm 0.34$ & & $3.0 \pm 0.04$ & & $6.5 \pm 0.09$ & \\
\hline
\end{tabular}

ns = not significant; $P=0.05\left(^{*}\right) ; P=0.01\left(^{(*)}\right) ; P=0.001(* * *)$

$\mathrm{ND}=$ not determined.

$\delta=$ Plant material stored for 16 years.

\# = Plant material stored for 12 years. 


\section{Antioxidant properties}

The effect of long-term storage on the radical scavenging activity of 21 plant materials is presented in Table 3. The lower the $\mathrm{EC}_{50}$ value, the higher the antioxidant activity index and the free radical scavenging activity. At $100 \mu \mathrm{g} / \mathrm{ml}$ concentration, the radical scavenging activity of all stored plant materials (with the exception of Protorhus longifolia) was either significantly higher or not different when compared to the freshly harvested materials. A comparison based on the $\mathrm{EC}_{50}$ values and antioxidant activity indices revealed a significantly higher radical scavenging activity in $58 \%$ of the stored plant materials. With the exception of $A$. oppositifolia and B. salviifolia (leaves), the radical scavenging activity of the remaining stored plant materials based on their $\mathrm{EC}_{50}$ values was not significantly different when compared to the fresh materials. The DPPH radical acts as both the probe and oxidant by accepting electrons from antioxidant compounds in the extract. There is a direct correlation between degree of hydroxylation of the bioactive compounds and DPPH radical scavenging activity [11]. Potent DPPH radical scavenging activities of medicinal plants have also been reported in other studies [11,13,14]. However, the significance of the present study lies in the observed high DPPH radical scavenging activity of aqueous methanol extracts obtained from medicinal plant material after prolonged storage.

Table 4 presents the effect of long-term storage on the antioxidant activity of medicinal plant materials evaluated based on $\beta$-carotene bleaching model. The

Table 4 Effect of long-term storage on antioxidant activity based on $\beta$-carotene bleaching model and acetylcholinesterase inhibitory properties of 21 South African medicinal plants

\begin{tabular}{|c|c|c|c|c|c|}
\hline \multirow[t]{2}{*}{ Plant species } & \multirow[t]{2}{*}{ Plant part(s) } & \multicolumn{2}{|c|}{ Antioxidant activity (\%) at $200 \mu \mathrm{g} / \mathrm{ml}$} & \multicolumn{2}{|c|}{ AChE inhibition (\%) at $1.0 \mathrm{mg} / \mathrm{ml}$} \\
\hline & & Stored & Fresh & Stored & Fresh \\
\hline Acokanthera oppositifolia ${ }^{\delta}$ & Roots & $54.7 \pm 3.4 \mathrm{~ns}$ & $40.0 \pm 7.71$ & $81.0 \pm 12.11 \mathrm{~ns}$ & $80.5 \pm 1.99$ \\
\hline Artemisia afra & Aerial parts & $45.8 \pm 3.34 \mathrm{~ns}$ & $39.8 \pm 4.94$ & $83.2 \pm 2.28 \mathrm{~ns}$ & $89.6 \pm 7.44$ \\
\hline Artemisia afra ${ }^{\delta}$ & Aerial parts & $44.4 \pm 7.20 \mathrm{~ns}$ & $39.8 \pm 4.94$ & $89.8 \pm 0.57 \mathrm{~ns}$ & $89.6 \pm 7.45$ \\
\hline Buddleja salviifolia" & Leaves & $39.1 \pm 7.69 \mathrm{~ns}$ & $58.3 \pm 3.04$ & $64.9 \pm 11.42 \mathrm{~ns}$ & $72.5 \pm 10.17$ \\
\hline Buddleja salviifolia & Twigs & $58.0 \pm 3.92 \mathrm{~ns}$ & $53.8 \pm 8.22$ & $73.0 \pm 15.63 \mathrm{~ns}$ & $63.9 \pm 4.05$ \\
\hline Clausena anisata ${ }^{\#}$ & Leaves \& Twigs & $23.6 \pm 4.06 \mathrm{~ns}$ & $49.8 \pm 11.19$ & $77.0 \pm 6.86 \mathrm{~ns}$ & $82.2 \pm 3.74$ \\
\hline Cussonia spicata ${ }^{\#}$ & Leaves & $55.7 \pm 6.45 \mathrm{~ns}$ & $41.8 \pm 4.70$ & $72.1 \pm 12.6 \mathrm{~ns}$ & $86.5 \pm 5.56$ \\
\hline Dombeya rotundifolia ${ }^{\#}$ & Leaves & $51.8 \pm 4.13 \mathrm{~ns}$ & $58.9 \pm 1.40$ & $84.1 \pm 5.54 \mathrm{~ns}$ & $87.6 \pm 2.88$ \\
\hline Ekebergia capensis ${ }^{\delta}$ & Leaves \& Twigs & $93.5 \pm 7.05^{* *}$ & $52.1 \pm 4.97$ & $73.8 \pm 7.24 \mathrm{~ns}$ & $89.7 \pm 6.08$ \\
\hline Leonotis intermedia $\delta$ & Leaves & $32.6 \pm 5.34 *$ & $52.9 \pm 4.09$ & $68.8 \pm 3.12 *$ & $87.8 \pm 3.83$ \\
\hline Leonotis leonurus ${ }^{\delta}$ & Leaves & $40.8 \pm 2.32 \mathrm{~ns}$ & $58.6 \pm 7.13$ & $78.1 \pm 3.67 \mathrm{~ns}$ & $73.2 \pm 0.43$ \\
\hline Merwilla plumbea ${ }^{\delta}$ & Bulbs & $57.0 \pm 6.42 \mathrm{~ns}$ & $45.1 \pm 4.06$ & $58.7 \pm 6.52 \mathrm{~ns}$ & $81.5 \pm 2.11$ \\
\hline Ocotea bullata & Bark & $57.8 \pm 7.33 \mathrm{~ns}$ & $62.3 \pm 8.83$ & $84.8 \pm 3.98 \mathrm{~ns}$ & $87.1 \pm 2.63$ \\
\hline Olea europaea & Leaves & $48.8 \pm 2.84 \mathrm{~ns}$ & $48.2 \pm 0.59$ & $69.2 \pm 5.99 \mathrm{~ns}$ & $85.4 \pm 3.39$ \\
\hline Pittosporum viridiflorum ${ }^{\#}$ & Leaves \& Twigs & $62.9 \pm 6.65 \mathrm{~ns}$ & $39.1 \pm 6.80$ & $96.2 \pm 0.71 \mathrm{~ns}$ & $70.5 \pm 8.36$ \\
\hline Plumbago auriculata ${ }^{\delta}$ & Leaves & $62.2 \pm 10.87 \mathrm{~ns}$ & $52.8 \pm 1.99$ & $82.3 \pm 5.54 \mathrm{~ns}$ & $87.3 \pm 2.20$ \\
\hline Protorhus longifolia ${ }^{\delta}$ & Leaves & $90.9 \pm 8.88 \mathrm{~ns}$ & $72.9 \pm 2.62$ & $51.8 \pm 9.07 \mathrm{~ns}$ & $40.07 \pm 2.60$ \\
\hline Solanum mauritianum ${ }^{\delta}$ & Leaves & $38.9 \pm 10.07 \mathrm{~ns}$ & $49.4 \pm 4.92$ & $78.5 \pm 5.84 \mathrm{~ns}$ & $85.9 \pm 3.94$ \\
\hline Spirostachys africana ${ }^{\#}$ & Leaves \& Twigs & $62.1 \pm 4.40 \mathrm{~ns}$ & $58.3 \pm 3.24$ & $90.4 \pm 5.57 \mathrm{~ns}$ & $82.4 \pm 3.51$ \\
\hline Synadenium cupulare $e^{\delta}$ & Leaves & $54.5 \pm 5.06 \mathrm{~ns}$ & $45.3 \pm 2.04$ & $75.3 \pm 4.07 \mathrm{~ns}$ & $81.1 \pm 2.77$ \\
\hline Tetradenia riparia ${ }^{\delta}$ & Leaves & $67.2 \pm 4.89 \mathrm{~ns}$ & $64.5 \pm 8.38$ & $80.8 \pm 1.73 *$ & $65.4 \pm 4.85$ \\
\hline Trichilia dregeana & Leaves \& Twigs & $65.2 \pm 7.46 \mathrm{~ns}$ & $50.6 \pm 8.81$ & $94.8 \pm 2.82 *$ & $81.1 \pm 3.99$ \\
\hline Ziziphus mucronata & Leaves & $54.5 \pm 3.65 \mathrm{~ns}$ & $42.6 \pm 6.62$ & $84.8 \pm 6.78 \mathrm{~ns}$ & $87.2 \pm 10.04$ \\
\hline Ziziphus mucronata & Leaves & $24.1 \pm 11.13 \mathrm{~ns}$ & $42.6 \pm 6.62$ & $90.4 \pm 4.09 \mathrm{~ns}$ & $87.2 \pm 10.04$ \\
\hline Galanthamine & & & & $84.1 \pm 1.45$ & \\
\hline Butylated hydroxytoluene & & $94.5 \pm 1.71$ & & & \\
\hline
\end{tabular}

$\mathrm{ns}=$ not significant; $P=0.05\left(^{*}\right) ; P=0.01\left(^{* *}\right) ; P=0.001\left({ }^{* * *}\right)$

$\delta=$ Plant material stored for 16 years.

\# = Plant material stored for 12 years.

Galanthamine $(20 \mu \mathrm{M})$ was used as a positive control in acetylcholinesterase assay. 
$\beta$-carotene bleaching assay simulates the oxidation of membrane lipid components and measures antioxidant activity towards linoleic acid [16]. The antioxidant activity of $E$. capensis stored plant material was significantly higher (almost two-fold) compared to the fresh material. On the other hand, the antioxidant activity of $L$. intermedia fresh plant material was significantly higher than that of the stored materials. With the exception of E. capensis and L. intermedia, there were no significant differences between the antioxidant activities recorded in both the stored and fresh plant materials. The retention of antioxidant activity in stored plant material suggests the stability of bioactive chemicals during prolonged storage. The detected bioactivity in the stored plant material provides interesting prospects in the future development of stable food additive compounds. In previous studies, high antioxidant activity from polar extracts of some plants has been attributed to hydrogendonating phenolic compounds and flavonoids $[2,16]$. However, the identification of specific phenolic compounds responsible for the high antioxidant activity of long-term stored plant materials remains a challenge for future research.

\section{Acetylcholinesterase inhibition activity}

Table 4 presents the effect of long-term storage on AChE inhibitory properties of the evaluated plant materials. Stored plant materials of $T$. riparia and $T$. dregeana showed a significantly higher AChE inhibition than the fresh ones. There was no significant difference between the percentage AChE inhibition by the stored and fresh materials of the remaining plant species. In general, the evaluated plant species exhibited high AChE inhibitory activity. Interestingly, medicinal plant materials retained AChE inhibitory activity even after prolonged storage (12 or 16 years). The results of the present study confirm the therapeutic value of stored medicinal plants in the pharmacotherapy of AD disease. The AChE inhibitory properties of plant-derived extracts obtained from freshly harvested material have been previously reported [16,32]. Recent studies have demonstrated a direct association between $\mathrm{AD}$ and antioxidant activity [16]. However, this is the first report on the antioxidant and AChE inhibitory properties of long-term stored medicinal plants. The present findings are important for traditional systems which are characterised by an holistic approach to health provision, based on the prophylactic properties of medicinal plants [6].

\section{Conclusions}

The current study presents evidence that dried medicinal plants stored under dark conditions at room temperature remain biologically active after long-term storage. Extracts of the stored plant material still exhibited potent antioxidant and AChE-inhibitory properties. These findings are significant as some medicinal plants may be utilised long after their time of harvesting. In addition, the prevention strategies practised in the Ayurvedic, Chinese and African medicinal systems often involve regular intake of medicinal plant extracts and/or herbal preparations, which are responsible for counteracting the oxidative stress effects caused by ROS. The high antioxidant activity and stability of the bioactive compounds in these medicinal plants offer interesting prospects for the identification of novel principles for application in food and pharmaceutical formulations. However, in vitro and in vivo safety evaluation of the stored medicinal plants is required.

\section{Abbreviations}

ACh: Acetylcholine; AChE: Acetylcholinesterase; AD: Alzheimer's disease; AAl: Antioxidant activity index; BHT: Butylated hydroxytoluene; CE: Catechin equivalents; DPPH: 2,2-diphenyl-1-picrylhydrazyl; DTNB: 5,5-dithiobis-2nitrobenzoic acid; DW: Dry weight; GAE: Gallic acid equivalents;

HE: Harpagoside equivalents; RSA: Radical scavenging activity; ROS: Reactive oxygen species.

\section{Competing interests}

The authors declare that they have no competing interests.

\section{Acknowledgements}

We are grateful to Mrs A. Young of University of KwaZulu-Natal Botanical Garden, as well as Dr M.E. Light and Mr B. Ncube of the Research Centre for Plant Growth and Development for assistance in the collection of plant material. The University of KwaZulu-Natal and National Research Foundation provided financial support.

\section{Authors' contributions}

SOA participated in the collection of plant materials, study design, extraction, conducting the assays, statistical analysis and drafting the manuscript. AOA was involved in the collection of plant materials, study design, extraction, carrying out the assays and editing the manuscript. MM participated in the collection of plant materials, study design, extraction, conducting the assays and drafting the manuscript. JVS coordinated the storage of plant materials and revised the manuscript. All authors read and approved the final manuscript.

Received: 3 April 2012 Accepted: 7 July 2012

Published: 7 July 2012

\section{References}

1. Bouayed J, Piri K, Rammal H, Dicko A, Desor F, Younos C, Soulimani R: Comparative evaluation of the antioxidant potential of some Iranian medicinal plants. Food Chem 2007, 104:364-368.

2. Atmani D, Chaher N, Berboucha M, Ayouni $K$, Lounis $H$, Boudaoud $H$, Debbache N, Atmani D: Antioxidant capacity and phenol content of selected Algerian medicinal plants. Food Chem 2009, 112:303-309.

3. Hoozesmans JJM, Veerhuis $R$, Rozemuller JM, Eikelenboom P: Neuroinflammation and regeneration in the in the early stages of Alzheimer's disease pathology. Int J Dev Neurosci 2006, 24:157-165.

4. Ndhlala AR, Aremu AO, Moyo M, Amoo SO, Van Staden J: Acetylcholineterase inhibitors from plant sources: friends or foes? In Cholinesterase: Production, Uses and Health Effects. Edited by White CJ, Tait JE. New York: Nova; 2012, 67-98.

5. Howes MJ-R, Houghton PJ: Plants used in Chinese and Indian traditional medicine for improvement of memory and cognitive function. Pharmacol Biochem Behav 2003, 75:513-527.

6. Houghton PJ, Howes M-J, Lee CC, Steventon G: Uses and abuses of in vitro tests in ethnopharmacology: visualizing an elephant. J Ethnopharmacol 2007, 110:391-400. 
7. Wong C-C, Li H-B, Cheng K-W, Chen F: A systematic survey of antioxidant activity of 30 Chinese medicinal plants using the ferric reducing antioxidant power assay. Food Chem 2006, 97:705-711.

8. Surveswaran S, Cai Y-Z, Corke H, San H: Systematic evaluation of natural phenolic antioxidants from 133 Indian medicinal plants. Food Chem 2007, 102:938-953.

9. Lizcano LJ, Bakkali F, Ruiz-Larrea MB, Ruiz-Sanz J: Antioxidant activity and polyphenol content of aqueous extracts from Colombian Amazonian plants with medicinal use. Food Chem 2010, 119:1566-1570.

10. Roy N, Laskar RA, Sk I, Kumari D, Ghosh T, Begum NA: A detailed study on the antioxidant activity of the stem bark of Dalbergia sissoo Roxb., an Indian medicinal plant. Food Chem 2011, 126:1115-1121.

11. Awah FM, Uzoegwu PN, Ifeonu P, Oyugi JO, Rutherford J, Yao X, Fehrmann F, Fowke KR, Eze MO: Free radical scavenging activity, phenolic contents and cytotoxicity of selected Nigerian medicinal plants. Food Chem 2012, 131:1279-1286.

12. Dutta RK, Maharia RS: Antioxidant responses of some common medicinal plants grown in copper mining areas. Food Chem 2012, 131:259-265.

13. Inayatullah S, Prenzler PD, Obied HK, Rehman A, Mirza B: Bioprospecting traditional Pakistani medicinal plants for potent antioxidants. Food Chem 2012, 132:222-229

14. Sharififar F, Moshafi MH, Shafazand E, Koohpayeh A: Acetyl cholinesterase inhibitory, antioxidant and cytotoxic activity of three dietary medicinal plants. Food Chem 2012, 130:20-23.

15. Oh MH, Houghton PJ, Whang WK, Cho JH: Screening of Korean herbal medicines used to improve cognitive function for anti-cholinesterase activity. Phytomedicine 2004, 11:544-548.

16. Ferreira A, Proença C, Serralheiro MLM, Araújom MEM: The in vitro screening for acetylcholinesterase inhibition and antioxidant activity of medicinal plants from Portugal. J Ethnopharmacol 2006, 108:31-37.

17. Houghton PJ, Ren Y, Howes M-J: Acetylcholinesterase inhibitors from plants and fungi. Nat Prod Rep 2006, 23:181-189.

18. Mukherjee PK, Kumar V, Mal M, Houghton PJ: Acetlycholinesterase inhibitors from plants. Phytomedicine 2007, 14:289-300.

19. Adewusi EA, Moodley N, Steenkamp V: Medicinal plants with cholinesterase inhibitory activity: a review. Afr J Biotechnol 2010, 9:8257-8276.

20. Hutchings A, Scott AH, Lewis G, Cunningham AB: Zulu Medicinal Plants: An Inventory. Pietermaritzburg: University of Natal Press; 1996.

21. Jäger AK, Hutchings A, Van Staden J: Screening of Zulu medicinal plants for prostaglandin-synthesis inhibitors. J Ethnopharmacol 1996, 52:95-100.

22. Duncan CA, Jäger AK, Van Staden J: Screening of Zulu medicinal plants for angiotensin converting enzyme (ACE) inhibitors. J Ethnopharmacol 1999, 68:63-70.

23. McGaw $\sqcup$, Jäger AK, Van Staden J: Antibacterial, anthelmintic and antiamoebic activity in South African medicinal plants. J Ethnopharmacol 2000, 72:247-263.

24. Eloff $\mathrm{JN}$ : It is possible to use herbarium specimens to screen for antibacterial components in some plants. J Ethnopharmacol 1999, 67:355-360.

25. Makkar HPS: Quantification of tannins in tree foliage: A laboratory manual for the FAO/IAEA Co-ordinated research project on 'Use of Nuclear and Related Techniques to Develop Simple Tannin Assays for Predicting and Improving the Safety and Efficiency of Feeding Ruminants on Tanniniferous Tree Foliage'. Vienna, Austria: Joint FAO/IAEA Division of Nuclear Techniques in Food and Agriculture; 2000.

26. Levieille $\mathrm{G}$, Wilson $\mathrm{G}$ : In vitro propagation and iridoid analysis of the medicinal species Harpagophytum procumbens and H. zeyheri. Plant Cell Rep 2002, 21:220-225.

27. Singleton VL, Rossi JA Jr: Colorimetry of total phenolics with phosphotungstic acid reagents. Am J Enol Vitic 1965, 16:144-158.

28. Fawole OA, Ndhlala AR, Amoo SO, Finnie JF, Van Staden J: Antiinflammatory and phytochemical properties of twelve medicinal plants used for treating gastro-intestinal ailments in South Africa. $J$ Ethnopharmacol 2009, 123:237-243.

29. Zhishen J, Mengcheng T, Jianming W: The determination of flavonoid contents in mulberry and their scavenging effects on superoxide radicals. Food Chem 1999, 64:555-559.

30. Porter LJ, Hrstich LN, Chan BG: The conversion of procyanidins and prodelphinidins to cyanidin and delphinidin. Phytochemistry 1986, 25:223-230.
31. Inoue $\mathrm{KH}$, Hagerman AE: Determination of gallotannins with rhodanine. Anal Biochem 1988, 169:363-369.

32. Fawole OA, Amoo SO, Ndhlala AR, Light ME, Finnie JF, Van Staden J: Anti-inflammatory, anticholinesterase, antioxidant and phytochemical properties of medicinal plants used for pain-related ailments in South Africa. J Ethnopharmacol 2010, 127:235-241.

33. Scherer R, Godoy HT: Antioxidant activity index (AAl) by the 2,2-diphenyl1-picrylhydrazyl method. Food Chem 2009, 112:654-658.

34. Moyo M, Ndhlala AR, Finnie JF, Van Staden J: Phenolic composition, antioxidant and acetylcholinesterase inhibitory activities of Sclerocarya birrea and Harpephyllum caffrum (Anacardiaceae) extracts. Food Chem 2010, 123:69-76.

35. Eldeen IMS, Elgorashi EE, Van Staden J: Antibacterial, anti-inflammatory, anti-cholinesterase and mutagenic effects of extracts obtained from some trees used in South African traditional medicine. J Ethnopharmacol 2005, 102:457-464

36. Bate-Smith EC: Investigation of the chemistry and taxonomy of sub-tribe Quillajeae of the Rosaceae using comparisons of fresh and herbarium material. Phytochemistry 1965, 4:535-539.

37. Bate-Smith EC, Harborne JB: Differences in flavonoid content between fresh and herbarium leaf tissue in Dillenia. Phytochemistry 1971, 10:1055-1058.

38. Phillipson JD: Chemical investigations of herbarium material for alkaloids. Phytochemistry 1982, 21:2441-2456.

doi:10.1186/1472-6882-12-87

Cite this article as: Amoo et al:: Antioxidant and acetylcholinesteraseinhibitory properties of long-term stored medicinal plants. $B M C$ Complementary and Alternative Medicine 2012 12:87.

\section{Submit your next manuscript to BioMed Central and take full advantage of:}

- Convenient online submission

- Thorough peer review

- No space constraints or color figure charges

- Immediate publication on acceptance

- Inclusion in PubMed, CAS, Scopus and Google Scholar

- Research which is freely available for redistribution
C Biomed Central 